\title{
Iterative Self-Dual Reconstruction on Radar Image Recovery
}

\author{
Charles I. O. Martins ${ }^{1} \quad$ Daniela M. Ushizima $^{2, *} \quad$ Fátima N. S.Medeiros $^{1}$ \\ Francisco N. Bezerra ${ }^{3} \quad$ Regis C. P. Marques ${ }^{3}$ \\ Nelson D. A. Mascarenhas ${ }^{4}$ \\ ${ }^{1}$ Department of Teleinformatics Engineering, Federal University of Ceará, Fortaleza-CE, Brazil \\ ${ }^{2}$ Computational Research Division, Lawrence Berkeley National Laboratory, Berkeley-CA, USA \\ ${ }^{3}$ Federal Institute of Education, Science and Technology, Ceará, Fortaleza-CE, Brazil \\ ${ }^{4}$ Federal University of São Carlos, São Carlos-SP, Brazil \\ charlesiury@gmail.com, dushizima@lbl.gov, fsombra@ufc.br, \\ nivando_bezerra@yahoo.com, regismarques@cefet-ce.br, nelsonddc.ufscar.br
}

This paper is a draft of the original one, by IEEE 2009 Workshop on Applications of Computer Vision (WACV)

\begin{abstract}
Imaging systems as ultrasound, sonar, laser and synthetic aperture radar (SAR) are subjected to speckle noise during image acquisition. Before analyzing these images, it is often necessary to remove the speckle noise using filters. We combine properties of two mathematical morphology filters with speckle statistics to propose a signal-dependent noise filter to multiplicative noise. We describe a multiscale scheme that preserves sharp edges while it smooths homogeneous areas, by combining local statistics with two mathematical morphology filters: the alternating sequential and the self-dual reconstruction algorithms. The experimental results show that the proposed approach is less sensitive to varying window sizes when applied to simulated and real SAR images in comparison with standard filters.
\end{abstract}

\section{Introduction}

Synthetic Aperture Radar (SAR) images allow to monitor natural scenes on the Earth surface due to the proper characteristics of its imagery system, enabling image capturing regardless of solar illumination or weather conditions. Its spectral operation range allows to detect high transmission of the electromagnetic waves in the atmosphere, even in adverse atmospheric conditions (e.g. during precipitations or cloudy sky).

Image analysis of SAR relies on digital image processing techniques as speckle modeling and fltering. Several adaptive speckle removal algorithms address the image in terms of small windows over regions assumed to be uniform (constant gray levels) or textured (abrupt intensity changes). Theoretically, we could control smoothness by modeling the image for particular window sizes, but the estimation of such model parameters in the presence of speckle noise is signal-dependent and non-Gaussian [1].

The methods proposed by Lee [6], Frost et al.[3], Kuan et al. [5] are considered standard algorithms in speckle f ltering, therefore they are often reported in performance comparisons as in [2,9]. The popularity of these flters among remote sensing users resulted in adaptations of the flters to be applied to ultrasound B-scan images, for diagnosis quality improvement. Lee and Kuan f lters are widely used to suppress speckle and they present similar performance when evaluated using speckle-noise local statistics as mean and standard deviation in $\mathrm{fxed}$ sliding window. These f lters can preserve steep edges if the window size is properly chosen. Window size values as high as $11 \times 11$ compromise f ne details in the image and window size values as low as $3 \times 3$ implies in insuff cient speckle noise suppression on homogeneous areas. This is a drawback reported in many papers $[3,5,9,10,17]$, a trade-off between preserving edges and reducing the strength of the noise. Lopes et al. [10] minimized the drawbacks of Frost and Lee flters by exploiting local SAR image statistics and specifying different levels of homogeneity. In addition to $[4,10,9]$, we propose a multiscale scheme that maintains $f$ ne details for larger windows by using morphological f lters combined to Lee $\mathrm{f}$ lter [7].

Our scheme uses a sliding window, which incorporates local statistics, adapting the Lee flter to include a combination of self-dual reconstruction and the alternating sequential $\mathrm{f}$ lters. The self-dual $\mathrm{flter}$ uses the median $\mathrm{f}$ lter to obtain marker images for the fltering process. The major contribution of our algorithm is to be fairly insensitive to the choice of the window size in comparison with the Lee $\mathrm{f}$ lter. In addition, we can easily modify the proposed scheme to include other standard speckle flters (e.g. Frost and Kuan) 
to generate marker images. We compare the proposed algorithm with the standard Lee f lter quantitatively by using the beta-coeff cient and the equivalent number of looks measures, showing that our algorithm outperforms the standard Lee flter.

Section 2 reports previous work in speckle noise modeling and fltering. Section 3 describes the proposed iterative self-dual reconstruction method and evaluates its performance in comparison with the standard formulation in [7]. In Section 4, we present experimental results by using simulated and real SAR images to summarize the advantages and contributions of the novel scheme in Section 5.

\section{Speckle Model and Filtering}

Two approaches can be adopted to reduce the speckle noise in SAR image: multilook processing and fltering techniques. The former improves the SAR image quality by averaging uncorrelated images from nonoverlapping spectra, consequently producing spatial resolution losses. We adopt the latter approach, which suppresses the speckle noise after the one-look image has been formed. Speckle noise can be described in terms of a multiplicative model $[7,8]$, given by $z=x . n$, where $z$ describes the amplitude of the noisy observed pixel at the position in linear detection, $x$ is the original signal and $n$ is the noise with unitary mean. The random variables $x$ and $n$ are assumed to be independent.

In the absence of a precise model for the original signal $x$, the noisy version of the signal is used to estimate the $a$ priori mean $\bar{x}$ and variance $\sigma_{x}^{2}$ of the original signal from the local mean $\bar{z}$, and local variance $\sigma_{z}^{2}$ in a $5 \times 5$ window [8]. In other words, the local mean is $\bar{z}=\bar{x} \cdot \bar{n}$ and the estimated variance is $\hat{\sigma}_{x}^{2}=\frac{\sigma_{z}^{2}-\sigma_{n}^{2} \bar{z}^{2}}{1+\sigma_{n}^{2}}$. The $\hat{\sigma}_{x}^{2}$ is the estimated variance of the original image and $\bar{n}$ is the unitary mean of the speckle noise. The speckle noise variance, $\sigma_{n}^{2}$, is an important parameter considered in designing speckle flters $[7,10]$. It measures the speckle strength, and it can be determined over featureless areas [8] by $\sigma_{n}^{2}=\frac{\sigma_{z}^{2}}{\bar{z}_{z}^{2}}$.

Lee [6] developed a widely used local linear minimum square error flter which is derived from the speckle model. This flter assumes that speckle is a random variable following a multiplicative noise with unitary mean, where $\hat{x}$ is the minimum mean square estimate of $x$. The noisy pixel is updated by the expression $\hat{x}=\bar{x}+k(z-\bar{z})$, where $\bar{x}$ is the local mean estimated on the sliding window, $z$ is the observed pixel and $k$ is the kernel of the f lter ranging between 0 and 1 , given by $k=\frac{\sigma_{x}^{2}}{\sigma_{x}^{2}+\bar{z} \sigma_{n}^{2}}$.

To perform speckle noise fltering, local statistics are computed over a fxed neighborhood (e.g. $5 \times 5$ window). Usually, the larger the window size, the lesser the performance of the speckle f lters concerning edge and $\mathrm{f}$ ne details preservation.
The proposed f lter scheme considers mathematical morphology techniques, which here unfolds in three main principles: alternating sequential flter (ASF), self-dual reconstruction and the Lee flter. This approach leads to the Iterative Reconstruction from Lee flter (IRLee), capable of overcoming imprecisions when edges and f ne detail preservation are necessary.

\subsection{Alternating Sequential Filter}

ASF is a sequence of alternate opening $(\gamma)$ and closing $(\phi)$ operations for windows of increasing size. These flters transform dark and bright regions differently depending on the initial operation: an opening or a closing to start the $\mathrm{fl}$ tering process. An ASF of order $i$ is a composition following one of the 4 different forms: $M_{i}=m_{i} m_{i-1} \ldots m_{2} m_{1}$, $R_{i}=r_{i} r_{i-1} \ldots r_{2} r_{1}, N_{i}=n_{i} n_{i-1} \ldots n_{2} n_{1}, S_{i}=$ $s_{i} s_{i-1} \ldots s_{2} s_{1}$, with $m_{i}, n_{i}, r_{i}, s_{i}$ def ned in terms of opening and closing operators as $m_{i}=\gamma_{i} \phi_{i}, r_{i}=\phi_{i} \gamma_{i} \phi_{i}$, $n_{i}=\phi_{i} \gamma_{i}, s_{i}=\gamma_{i} \phi_{i} \gamma_{i}$. The parameter $i$ represents the size of the window for morphological f ltering.

An ASF cannot be self-dual due to the inherent asymmetry in its def nition $N i \neq M i$ [13]. This technique is widely used for f ltering radar images [14], but their f nal result depends on the initial operator to start the sequence of transformations with no guarantee of preservation of thin structures in the presence of strong noise level. A self-dual flter [16] could be applied to overcome initial condition dependence. This motivated the use of self-dual reconstruction to avoid such an initial operator dependency and to preserve thin details. The self-dual morphological reconstruction is reviewed in the next section.

\subsection{Self-dual Reconstruction}

Morphological reconstruction by dilation (or erosion) is an operator that removes dark (or bright) regions from a marker image constrained by a mask image. Particularly, self-dual reconstruction combines reconstruction by using dilation and erosion to achieve the same treatment to dark and bright regions of the image. The self-dual reconstruction $R_{g}^{\nu^{\prime}}(f)$ of a marker image $f$ constrained by a mask image $g$ is def ned by:

$$
\left[R_{g}^{\nu^{\prime}}(f)\right](\mathrm{x})=\left\{\begin{array}{l}
{\left[R_{g}^{\delta}(f \wedge g)\right](\mathrm{x}), \text { if } f(\mathrm{x}) \leq g(\mathrm{x})} \\
{\left[R_{g}^{\varepsilon}(f \vee g)\right](\mathrm{x}), \text { otherwise }}
\end{array}\right.
$$

where $R^{\delta}$ and $R^{\varepsilon}$ correspond to the morphological reconstruction by dilation and erosion, respectively.

Self-dual or self-complementary morphological flters have proven to be useful in speckle noise reduction as in [16], especially on radar data, where there is no clear distinction between background and foreground in the images and motivated several other works in noise fltering. Selfdual operators are based upon the assumption that the tar- 
geted image structures are image extrema [15], not considering statistical properties, assumption that neglects the multiplicative model of the speckle noise.

A common approach to remove speckle noise is the use of the original noisy image as a mask, while a marker image is obtained by processing the original one with a median $\mathrm{fl}$ ter, which does not incorporate any speckle statistics [15]. The median flter removes noise and, subsequently, the reconstruction attempts to restore degraded $\mathrm{f}$ ne details but it fails at those entirely removed by the flter.

We noticed that thin structures could be preserved in the fltering process when combining multiscale parameters to the reconstruction process. We design a novel scheme that a set of Lee flters of increasing window size, as a sequential flter, with self-dual reconstruction to obtain speckle removal and preservation of thin details of the image. The closing and opening operations in the ASF are substituted by the Lee flter to smooth the image, while the speckle statistics component guarantees the preservation of image details during the reconstruction process as it follows.

\section{Methodology}

Our method adapts the Lee flter with morphological reconstruction by performing adequate noise removal with image statistics, and maintaining f ne image details. We propose that the window size increases in each iteration according to the relation: $W_{n}=W_{1}+2 \times(n-1)$, for $W_{1}$ corresponding to the minimum window size $(3 \times 3)$ and $n$ $(n \geq 1)$ corresponds to the number of the current iteration. Thus, the second iteration generates a Lee fltered marker image with a $5 \times 5$ window $\left(W_{2}\right)$, the third with a $7 \times 7$ window $\left(W_{3}\right)$, the fourth with a $9 \times 9$ window $\left(W_{4}\right)$ and so on.

The implemented algorithm denoted Iterative Reconstruction from Lee (IRLee) flter of order $n$ is described as follows:

$$
\operatorname{IRLee}_{n}(f)=\left\{\begin{array}{l}
R_{L_{1}(f)}(f), \text { for } n=1 \\
R_{L_{n-1}\left(I R L e e_{n-1}(f)\right)}(f), \text { for } n>1
\end{array}\right.
$$

where $L_{n}(f)$ denotes the Lee flter applied to an image $f$ using a window of size $W_{n}$.

The IRLee fltering starts by considering the $I R L e e_{0}$ image to be the original one (speckled image), then the Lee flter is applied to the IRLee $e_{n-1}$ image, generating a marker image $L_{n}$ and the f nal $I R L e e_{n}$ image is obtained by the reconstruction process using the original image as a mask. Here, instead of estimating the standard deviation of the speckle noise $\left(\sigma_{n}\right)$ in each IRLee image we keep it constant for every iteration. We assume that some pixel values in each iteration are still affected by the multiplicative noise and therefore we can use the same $\sigma_{n}$ parameter for the Lee flter with a larger window. This assumption is mo- tivated by the self-dual reconstruction flter effect obtained when it is applied to the previous Lee fltered image. The self-dual reconstruction f lter modif es several pixel values turning them into values closer to their correspondents in the mask image.

The Lee's flter does not preserve f ne details when considering large windows, resulting in increased blurring effect over the marker images. The self-dual reconstruction minimizes this drawback by considering an iterative process that preserves f ne details, in spite of the blurring effect created in the Lee fltered marker images. Next sections use $I R L e e$ to refer to the flter whose marker images are generated by using Lee flter, and IRMedian when the marker images are generated by using the median flter.

\subsection{Speckle Filtering Validation}

We compared the proposed approach with other flters by considering measures related to edge preservation and speckle strength reduction. We demonstrate the eff cacy of the IRLee f lter by calculating the standard deviation to the mean ratio $\left(\beta_{w}\right)$, followed by the verif cation of the speckle noise strength in the fltered images, given by [8] $\beta_{w}=\frac{\sigma_{w}}{\mu_{w}}$.

The sample mean $\left(\mu_{w}\right)$ and standard deviation $\left(\sigma_{w}\right)$ can be estimated over a window $w$ comprising $N$ pixels in a homogeneous area of the fltered SAR image.

The equivalent number of looks $(E N L)$ after fltering provides a quantitative evaluation of the degree of speckle smoothing [9], supposing that an ideal flter would give an inf nite $E N L$ for a plain homogeneous area. This measure over homogeneous areas also accounts for the speckle noise strength and is def ned for amplitude SAR images in [8] as $E N L=\left(\frac{0.5227}{\beta_{w}}\right)^{2}$, where 0.5227 is the value of the $\sigma_{n}$ for an amplitude one-look SAR image.

The beta coeff cient or speckle index represented by $\beta_{w}$ and $E N L$ are useful measures to evaluate speckle noise $\mathrm{fl-}$ ters. In addition, we also evaluate edge preservation by using the $A$ coeff cient, namely [12]

$$
A=\frac{\Gamma(\Delta S-\overline{\Delta S}, \widehat{\Delta S}-\overline{\widehat{\Delta S}})}{\sqrt{\Gamma(\Delta S-\overline{\Delta S}, \Delta S-\overline{\Delta S}) \cdot \Gamma(\widehat{\Delta S}-\overline{\widehat{\Delta S}}, \widehat{\Delta S}-\overline{\widehat{\Delta S}})}}
$$

where $\Delta S$ and $\widehat{\Delta S}$ are the high-pass fltered versions of an original image $(S)$ and the denoised one $(\hat{S})$, respectively, obtained with a $3 \times 3$ pixel standard approximation of the Laplacian operator and the function $\Gamma\left(S_{1}, S_{2}\right)=$ $\sum_{i=1}^{K} S_{1 i} \cdot S_{2 i}$

\section{Experimental Results}

This section describes the image processing results by running the proposed morphological speckle f lter using artif cial and real SAR images. We evaluated the results using 


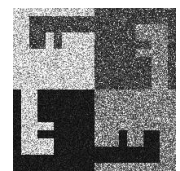

(a)

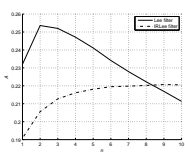

(b)
Figure 1. (a) Simulated 3.0 looks SAR image; (b) $A$ values calculated to images processed by the Lee flter using a window of increasing size (solid) and processed by IRLee flter with an increasing amount of iterations (dashed).

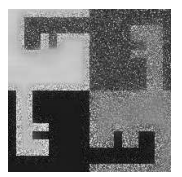

(a)

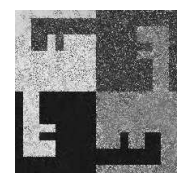

(b)
Figure 2. Blurring effect observed for $n=5$ by applying (a) the median flter and (b) the Lee flter to the image in Figure 1a to obtain the mask images for the IRLee flter.

Lee flter as in $[7,8]$ and its major modif cation, the proposed algorithm IRLee by measuring the $\beta_{w}$ and $E N L$ to evaluate the speckle noise reduction and the $A$ coeff cient measure edge preservation. Due to lack of real segmentation of the SAR images, we calculated the $A$ coeff cient only for a synthetic image. The correlation measure, $A$, should be close to unity for an optimal edge preservation effect whereas low values of $\beta_{w}$ (close to zero) in homogeneous areas imply low speckle f uctuations.

Figure $1 \mathrm{~b}$ displays the $A$ values calculated for the processed images using the Lee and IRLee f lters over the f1tered versions of the noisy image in Figure 1a. In Figure $1 \mathrm{~b}$, the curve of $A$ values for the Lee flter shows that the edges are not preserved ( $A$ tends to values close to zero) as the window's size increases. This is an evidence that the use of the Lee flter with an analyzing window with dimensions greater than $5 \times 5$ does not guarantee edge preservation. This effect is not observed for the IRLee flter in the same graphic, where the $A$ values asymptotically approximate $A=0.22$ as the number of iterations of the proposed algorithm increases. It implies that the blurring effect over the homogeneous areas increases but the edge smearing is stable. This effect appears in Figure 2 to demonstrate the good performance of the proposed technique over the standard method, according to edges preservation while reducing speckle noise.

Figure 3a presents an amplitude (square root of the intensity) SAR image over Thetford, England, which was obtained by the Canadian airborne C-SAR in slant-range projection. It is a nominal 7-looks image, and the estimated number of looks $(E N L)$, used in the forthcoming tests, was of $6.7, \mathrm{VV}$ polarization and spatial resolution of $6 \mathrm{~m}$.

In Figure $3 b$, we present the $\beta_{w}$ values calculated for the processed images using the Lee and IRLee flters over the image in Figure 3a. The fltering process ended up with an analyzing window comprising $21 \times 21$ pixels, or ten iterations. The $\beta_{w}$ values were calculated in a $15 \times 15$ window $(W)$ over a homogeneous area of the original image and its corresponding fltered versions. Figure 4 displays the results of the proposed algorithm when using the median and Lee f lters to obtain marker images for the IRLee fltering process. We can visually observe from these results that our approach outperformed the one which uses the standard median flter. The same results can be observed when applying these two methods to the image in Figure 5a, where the blurring effect on the processed images can be observed in Figure 6.

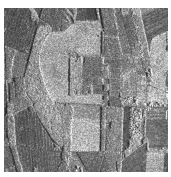

(a)

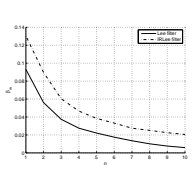

(b)
Figure 3. (a)Original 6.7 looks SAR image; (b) $\beta_{w}$ values calculated to images processed by the Lee flter using a window of increasing size (solid) and processed by the IRLee flter with an increasing amount of iterations (dashed).

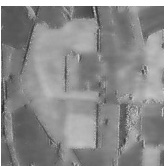

(a)

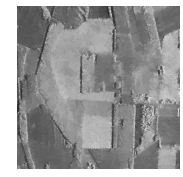

(b)
Figure 4. Blurring effect observed for $n=5$ by applying (a) the median and (b) the Lee flter flter to the image in Figure $3 a$ to obtain the marker images for the IRLee f lter.

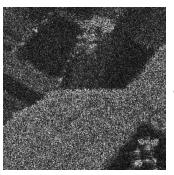

(a)

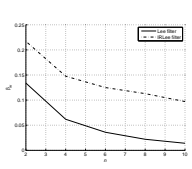

(b)
Figure 5. (a) Original one-look SAR image; (b) $\beta_{w}$ values calculated to images processed by the Lee flter using an window of increasing size (solid) and processed by IRLee flter with an increasing amount of iterations (dashed).

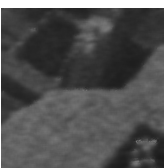

(a)

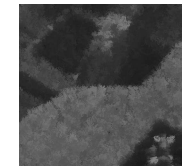

(b)
Figure 6. Blurring effect observed for $n=5$ by applying (a) the median flter and (b) the Lee flter to the image in Figure $5 \mathrm{a}$ to obtain the marker images for the IRLee f lter. 
The $\beta_{w}$ values, observed in Figures $3 \mathrm{~b}, 5 \mathrm{~b}$, decrease exponentially as the window size, or the number of iterations, increases in the two methods. However, the $\beta_{w}$ values (solid lines) obtained from the Lee fltered images indicate that homogeneous areas tend to be created more quickly when using only this standard flter. These values are lower than the ones obtained on the IRLee fltered images (dashed lines). Thus, using a greater window size (e.g. $9 \times 9)$ in the Lee f $1-$ ter features such as edges and f ne details will be vanished insofar as the speckle noise will be suppressed. This effect can be observed in Figures 4 and 6, which illustrate the increasing blurring effect on the images fltered with the standard Lee f lter. Differently, the Iterative Reconstruction from Lee flter (IRLee) suppresses speckle noise while effectively preserves image features (edges) with increasing fltering window size.

Comparative experiments obtained for the IRLee flter also included the real SAR image displayed in Figure 7a. It was acquired by the JERS-1 satellite over the Tapajos National Forest, Para, Brazil, with spatial resolution of $18 \mathrm{~m}$, nominal look angle of 35 degrees and 3-looks (nominal).

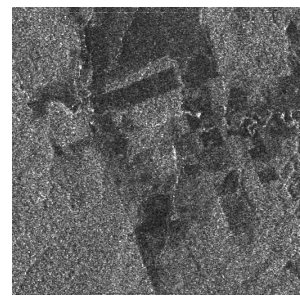

(a)

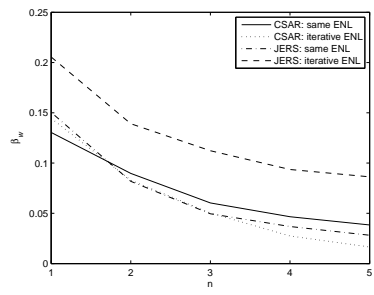

(b)
Figure 7. (a) JERS image taken over the Tapajos National Forest, in Brazil; (b) $\beta_{w}$ values calculated to IRLee fltered images by using the Lee flter parameter $\sigma_{n}$ estimated in each iteration and by using $\sigma_{n}$ constant in all iterations.

Figure $7 \mathrm{~b}$ illustrates the behavior of the IRLee flter when estimating the equivalent number of looks in the iterated fltered SAR images (Figure $3 a$ and Figure 7a) and when using the same $E N L$ in all iterations. These results motivated us to adopt $\sigma_{n}$ constant over all iterations for comparison purposes. In [11] Oliver and Quegan reported that diff culty in estimating the equivalent number of looks in iterated fltered images where $E N L$ is expected to increase as iterations proceed. According to the authors the estimation becomes diff cult as it varies across the image.

Figure 8a displays a 4-looks RADARSAT-1 image acquired in the ScanSAR wide mode, swath width of $150 \mathrm{~km}$ and polarization $\mathrm{HH}$, radar incidence angle of $27^{\circ}$ and with $12.5 \mathrm{~m}$ spatial resolution, corresponding to a region of the North coast of Rio Grande do Norte (RN), Brazil. In this image the white square encompasses bright points over the upper target on the right side which consist of oil platforms in the ocean. Figure $8 \mathrm{~b}$ corresponds to a detail image which contains small targets as oil platforms in the ocean, to be preserved in the fnal result due to their importance in the image interpretation. Figures $8 \mathrm{c}$ and $8 \mathrm{~d}$ present the results using the median and Lee flters, respectively, to obtain the marker images for the iterative process. Figure 9 quantif es the ability of our method to preserve and enhance details while reducing the noise. Figure 9 depicts an image profle from the target on the right of images presented in Figures $8 \mathrm{c}$ and $8 \mathrm{~d}$. These patterns show that the blurring effect applied to the original image (solid line) using the median flter to generate the markers (IRMedian - dashed line) attenuates the pattern more than the Lee flter in the method. According to these results, targets are better preserved using our method (IRLee in dash-dotted line), as Figure 8d displays. The top-left targets almost disappeared from Figure $8 \mathrm{c}$, demonstrating that targets vanish when the marker image does not take into account the statistical model of the speckle noise.

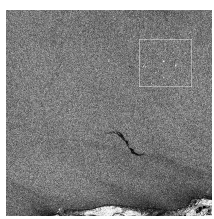

(a)

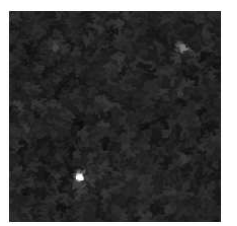

(c)

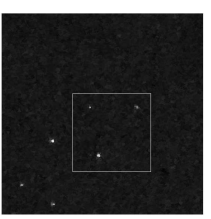

(b)

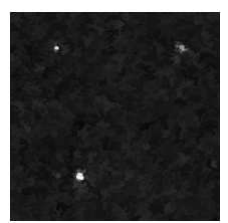

(d)
Figure 8. (a) Original 4-looks RADARSAT-1 image; (b) Oil platforms in the area inside the white square; (c) Filtered area inside the white square using the median $\mathrm{flter}$ to obtain the marker image for our method; (d) Filtered area inside the white square using the Lee $f$ lter to obtain the marker image for our method.

\section{Concluding Remarks}

We introduced the Iterative Reconstruction from Lee $\mathrm{f}$ 1ter (IRLee), a morphological iterative flter for SAR images, which takes into account the multiplicative model and speckle statistics. The proposed algorithm incorporates the multiplicative speckle model to the marker images, providing a more adequate morphological $f$ lter than the standard self-dual f lter with respect to edge enhancement and preser-

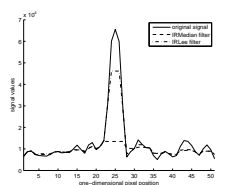

Figure 9. Image prof le from the target illustrate on the right of Figures $8 \mathrm{c}$ and $8 \mathrm{~d}$. 
vation. An ideal speckle filte should reduce noise while preserving edges and fin details. The standard Lee's fil ter can reduce speckle effects but it also smears edges. The IRLee algorithm is an effective filte in reducing speckle noise from uniform areas and in enhancing and preserving edges, enabling larger windows to be used, with consequent lower impact on edges. Note that although we have adopted the Lee filte to generate marker images, other speckle fil ters might be feasible. The comparative results between the Lee filte, the standard self-dual filte and the proposed scheme pointed out the importance of incorporating speckle statistics of SAR images to improve filterin in multiscale scenarios. We are currently investigating other filter to generate marker images at lower time computing.

\section{Acknowledgments}

This work was supported by $\mathrm{CNPq}$ and the Applied Mathematical Science subprogram of the Offic of Energy Research, U.S. Department of Energy, under Contract Number DE-AC02-05CH11231.

\section{References}

[1] K. Eom. Robust facet model for application to speckle noise removal. In Int. Conf. on Pattern Recognition (ICPR04), pages 695-698, 2004.

[2] A. C. Frery and S. J. S. Sant'anna. Non-adaptive robust fil ters for speckle noise reduction. In Proc. of IEEE Brazilian Symp. on Comput. Graph. and Image Process., pages 165174, October 1993.

[3] V. S. Frost, J. A. Stiles, K. S. Shanmugan, and J. C. Holtzman. A model for radar images and its application to adaptive digital filterin of multiplicative noise. IEEE Trans. Pattern Anal. and Mach. Intell., 4:157-165, 1982.

[4] D. T. Kuan, A. A. Sawchuck, T. C. Strand, and P. Chavel. Adaptive noise smoothing filte for images with signaldependent noise. IEEE Trans. PAMI, 7:165-177, 1985.

[5] D. T. Kuan, A. A. Sawchuck, T. C. Strand, and P. Chavel. Adaptive restoration of images with speckle. IEEE Trans. Acoust., Speech, and Sig. Proc., 35:373-383, 1987.

[6] J. S. Lee. Digital image enhancement and noise filterin by use of local statistics. IEEE Trans. Pattern Anal. and Mach. Intell., 2:165-168, 1980.

[7] J. S. Lee. Speckle analysis and smoothing of synthetic aperture radar images. Comput. Graph. and Image Process., 17:24-32, 1981.

[8] J. S. Lee, I. Jurkevich, P. Wambacq, and A. Oosterlinck. Speckle filterin of synthetic aperture radar images: A review. Remote Sens. Rev., 8:313-340, 1994.

[9] A. Lopes, E. Nezry, R. Touzi, and Laur. Structure detection and statistical adaptive speckle in SAR mages. Int. J. of Remote Sens., 14(9):1735-1758, 1993.

[10] A. Lopes, R. Touzi, and E. Nezry. Adaptive speckle filter and scene heterogeneity. IEEE Geosci. and Remote Sens. Lett., 28(6):992-1000, November 1990.
[11] C. Oliver and S. Quegan. Understanding synthetic aperture radar images. Artech House, Boston, 1998.

[12] F. Sattar, L. Floreby, G. Salomonsson, and B. Lövström. Image enhancement based on a nonlinear multiscale method. IEEE Trans. Image Process., 6:191-197, 1997.

[13] J. P. Serra. Image Analysis and Mathematical Morphology, volume 2. Academic Press, Orlando, 2nd edition, 1988.

[14] S. Sigurjonsson, J. Benediktsson, J. Sveinsson, G. Lisini, and J. Chanussot. Street tracking based on SAR data from urban areas. In Proc. of IEEE IGARSS05, pages 1273-1276, 2005.

[15] P. Soille. Beyond self-duality in morphological image analysis. Image and Vision Comput., 23:249-257, 2005.

[16] P. Soille and M. Pesaresi. Advances in mathematical morphology applied to geoscience and remote sensing. IEEE Trans. Geosci. and Remote Sens., 40:2042-2055, 2002.

[17] G. Subrahmanyam, A. N. Rajagopalan, and R. Aravind. A recursive filte for despeckling SAR images. IEEE Trans. Image Process., 17(10):1969-1974, 2008. 